\title{
THE STELLAR SEISMOLOGY OF THE HOT WHITE DWARF STAR PG1159-035
}

\author{
Steven D. Kawaler \\ Yale University, Center for Solar and Space Research \\ P.O. Box 6666 \\ New Haven, CT 06511
}

\begin{abstract}
Asymptotic analysis of the equations of nonradial adiabatic oscillation shows that there is a "characteristic period spacing" for $g$-modes with the same degree $(l)$ and consecutive values of the radial wavenumber $(n)$. If modes with the same $l$ but different $n$ are present in a pulsating star, then comparison of the characteristic period spacing in appropriate stellar models with period differences between the observed pulsation periods can provide mode identifications, and thereby constrain other physical properties of the star. A simple empirical model of the observed period spectrum of the hot white dwarf star PG1159-035 yields two statistically significant mean period intervals of $21.0 \pm 0.3$ or $8.8 \pm 0.1 \mathrm{~s}$. We also evaluate the period interval for models of hot white dwarfs that are representative of PG1159 stars. The observed intervals correspond very closely to those derived from the theoretical models. This analysis indicates that the mass of PG1 159-035 is $0.60 \mathrm{M}_{\odot}$ and that it is either a dipole $(l=1)$ or $l=3$ pulsator (or both!), pulsating in high radial overtone $g$-modes.
\end{abstract}

\section{8 -MODE PERIOD SPACINGS AS SEISMOLOGICAL INDICATORS}

Most pulsating white dwarf stars (the PG1159 variables, DBV stars, and the ZZ Ceti stars) are multiperiodic $g$-mode pulsators. Tassoul (1980) shows that the periods of high radial overtone $(n) g$-modes with the same degree $l$ obey theasymptotic relation:

$$
\Pi_{n}-\Pi_{n_{0}} \approx\left(n-n_{0}\right) \Delta \Pi \text {, }
$$

where $\Pi_{n}$ is the period for a mode of order $n$, and no is a reference mode. The minimum period interval for a given $l, \Delta \Pi$, is equal to $\Pi_{0} \times[l(l+1)]^{-1 / 2}$, where the "characteristic period spacing" $\Pi_{0}$ is determined by pulsation analysis of stellar models. $\Pi_{0}$ can also be expressed as an integral over equilibrium model quantities to a high degree of accuracy, making it a convenient parameter with which to compare the expected adiabatic pulsation properties of different models and stars.

If a star is pulsating in many $g$-modes with the same value of $l$ but different values of $n$, then the period differences should all be integer multiples of a minimum period interval.

Any such statistically significant minimum period interval in the period spectrum of a real star allows the full power of the theoretical analysis of the adiabatic pulsation properties to make fundamental statements about the star and its pulsations. In this paper, we show how the Kolmogorov-Smimov test can be used to verify constant period intervals in the period spectra of rich pulsators. We employ this technique to show that the pulsating hot white dwarf PG1159-035 has two statistically significant period intervals. We then use these period intervals, combined with theoretical models, to show that it is pulsating in either $l=1$ or $l=3$ modes (or both), and has a mass of $0.60 \mathrm{M}_{\odot}$. 


\section{KOLMOGOROV-SMIRNOV TEST FOR SIGNIFICANT PERIOD SPACINGS}

An appropriate statistical test for the significance of a perceived uniform period spacing is provided by the Kolmogorov-Smirnov (K-S) test. Generalizing equation (1):

$$
\mathrm{r}_{\mathrm{i}}(\Delta \Pi)=\underline{\underline{n}}_{\mathrm{i}}-\operatorname{int}\left(\underline{n}_{\mathrm{i}}\right) \text {, }
$$

where $\operatorname{int}(\mathrm{x})$ is the greatest integer less than $\mathrm{x}$, and

$$
\underline{n}_{i}=\left(\Pi_{i}-\Pi_{\text {shortest }}\right) / \Delta \Pi
$$

If the periods all have the same value of $l$, then the $r_{i}(\Delta \Pi)$ will be zero for the correct value of $\Delta \Pi$. If the assumed value of $\Delta \Pi$ is incorrect, or the periods are not representable by equation (1), then $r_{i}(\Delta \Pi)$ will not in general be zero. For a random distribution of periods, $r_{i}(\Delta \Pi)$ will be a uniformly distributed random number between 0 and 1 .

The K-S test provides a measure of the probability that a set of numbers is drawn from a chosen distribution (see, for example, Press et al. 1986). Here, we use the test to determine the probability that the $r_{i}(\Delta \Pi)$ are from a uniform distribution for a given $\Delta \Pi$. In effect, we use the test in a backwards fashion: any uniform (or at least systematically non-random) period spacings present will appear as minima in $Q$, where $Q$ is the probability that the $r_{j}$ 's are randomly distributed. We say that a uniform period interval is signifcant at a "confidence level" of (1-Q) $\times 100 \%$.

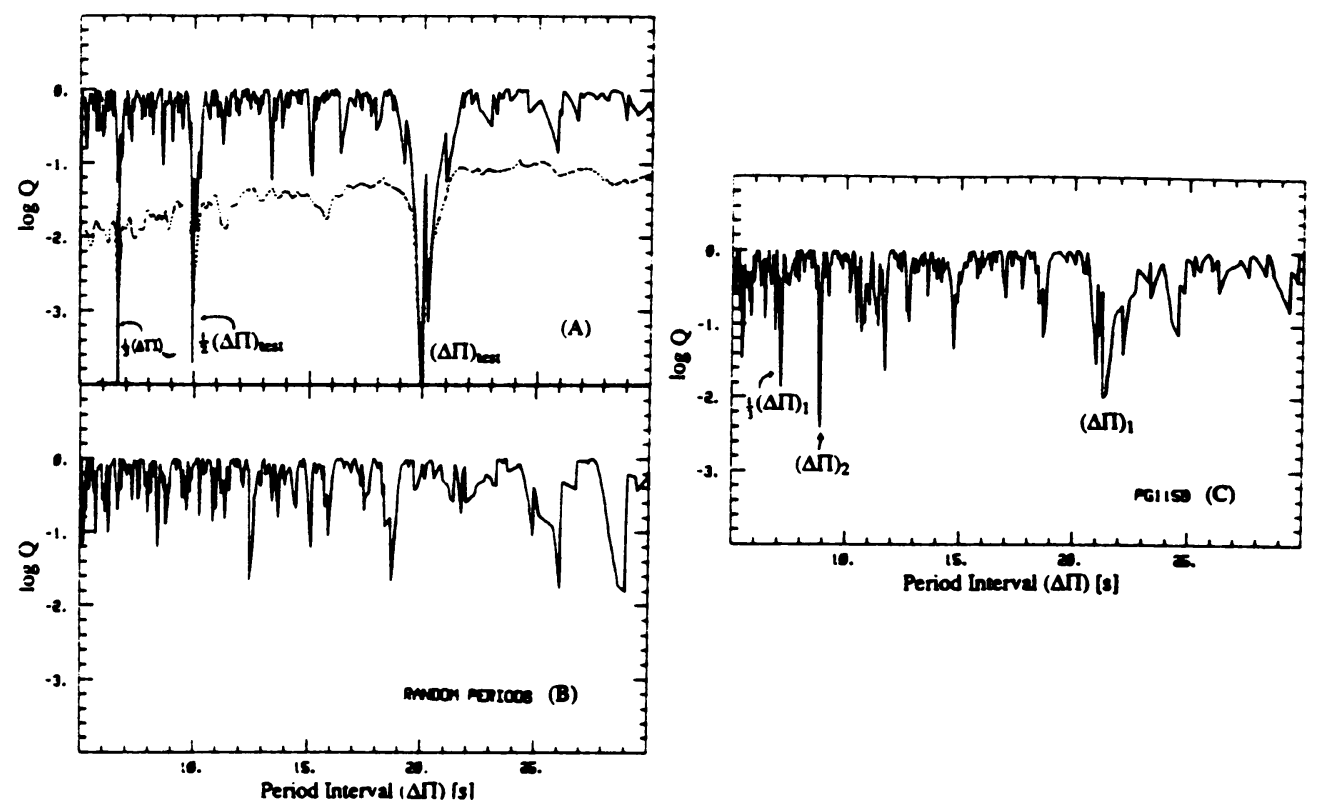

Eigure - 1:

(A) Results of the $K$-S test for an ideal set of 8 periods with period intervals of multiples of 20 seconds.

(B) $K$-S test with 8 random periods. Note that no period interval is above the $98.5 \%$ confidence level.

(C) $K$-S test applied to the 8 periods of PG1159-035 (see Table 1). The star shows two distinct period intervals: $8.82 \pm 0.07 \mathrm{~s}$ and $21.1 \pm 0.3 \mathrm{~s}$. 


\section{UNIFORM PERIOD INTERVALS IN THE HOT WHITE DWARF PG1159-035}

PG1159-035 pulsates in 8 modes with periods from 390 to 830 seconds (Winget $e$ t al. 1985), as indicated in Table 1. Application of the K-S test to this set of periods reveals two statistically significant period intervals of $21.1 \mathrm{~s}$ and $8.82 \mathrm{~s}$ (Figure 1C). The confidence level of the $21.1 \mathrm{~s}$ period spacing increases if the $424.4 \mathrm{~s}$ period is not used in the test; a similar enhancement of the $8.82 \mathrm{~s}$ spacing occurs if all but the $516.0 \mathrm{~s}$ period are used. In Table 1, we show that these spacings do indeed provide a simple description of the periods in PG1159 in terms of equation (1).

Table 1. THE PERIOD SPECTRUM OF PG1159-035

\begin{tabular}{lcccccc} 
& \multicolumn{2}{c}{$\Delta \Pi=21.1 \pm 0.3 \mathrm{~s}$} & & \multicolumn{2}{c}{$\Delta \Pi=8.82 \pm 0.07 \mathrm{~s}$} \\
\cline { 2 - 3 } \cline { 5 - 6 } $\begin{array}{l}\text { Observed } \\
\text { Period [s] }\end{array}$ & $n-n \mathrm{o}$ & $\Pi_{n \mathrm{o}}+\Delta \Pi(n-n \mathrm{o})$ & & $n-n \mathrm{o}$ & $\Pi_{n \mathrm{n}}+\Delta \Pi(n-n \mathrm{o})$ \\
$=========$ & $====$ & $==========$ & & $====$ & $=========$ \\
$390.0 \pm 0.9$ & 0 & $390.0 \pm 0.9$ & & 0 & $390.0 \pm 0.9$ \\
$424.4 \pm 1.1$ & $\ldots$ & $\ldots \ldots$ & & 4 & $425.3 \pm 0.3$ \\
$451.5 \pm 1.2$ & 3 & $453.3 \pm 1.2$ & & 7 & $451.7 \pm 0.5$ \\
$495.0 \pm 1.5$ & 5 & $495.5 \pm 1.5$ & & 12 & $495.8 \pm 0.8$ \\
$516.0 \pm 1.6$ & 6 & $516.6 \pm 1.7$ & & 14 & $513.5 \pm 1.0$ \\
$538.9 \pm 1.7$ & 7 & $537.7 \pm 2.0$ & & 17 & $539.9 \pm 1.2$ \\
$645.2 \pm 2.5$ & 12 & $643.2 \pm 3.1$ & & 29 & $645.8 \pm 2.0$ \\
$831.7 \pm 4.2$ & 21 & $833.1 \pm 5.3$ & & 50 & $831.0 \pm 3.5$
\end{tabular}

The corresponding values of the characteristic period spacing $\Pi_{0}$ for various possible values of 1 are:

\begin{tabular}{lccc} 
& $\Delta \Pi=21.1 \pm 0.3$ & $\Delta \Pi=8.82 \pm 0.07$ \\
\cline { 2 - 2 }$l=1$ & $29.8 \pm 0.4$ & & $12.5 \pm 0.1$ \\
$l=2$ & $51.7 \pm 0.9$ & & $21.6 \pm 0.2$ \\
$l=3$ & $73.1 \pm 1.2$ & & $30.6 \pm 0.2$ \\
$l=4$ & $94.4 \pm 1.6$ & & $39.4 \pm 0.3$
\end{tabular}

\section{COMPARISON WITH MODELS OF HOT WHITE DWARFS}

Models of evolving hot white dwarfs (Kawaler 1986) show that $\Pi_{0}$ is insensitive to luminosity and composition at $\mathrm{L}>10 \mathrm{~L}_{0}$; it is most sensitive to total stellar mass (Figure 2). A period spacing of $\Delta \Pi=21.1 \mathrm{~s}$ demands that if $P G 1159$ is a garden-variety hot white dwarf, then it is pulsating in $l=1$ modes and it has a mass of $0.6 \mathrm{M}$. This is clear from Figure 3, which shows that any other value of $l$ would require a mass for PG1159-035 that is very far from $0.6 \mathrm{M}_{0}$, the well-established mean mass of field white dwarfs (Weidemann and Koester 1983). A spacing of $\Delta \Pi=8.82 \mathrm{~s}$ also indicates a mass of $0.6 \mathrm{M}_{\odot}$ for PG 1159 if the modes are $l=3$ modes. It is possible that with this shorter period spacing the modes are either $l=2$ or $l=4$, but those values of $l$ would imply masses that are still at the tails of the white dwarf mass distribution of Weidemann and Koester (1983). 


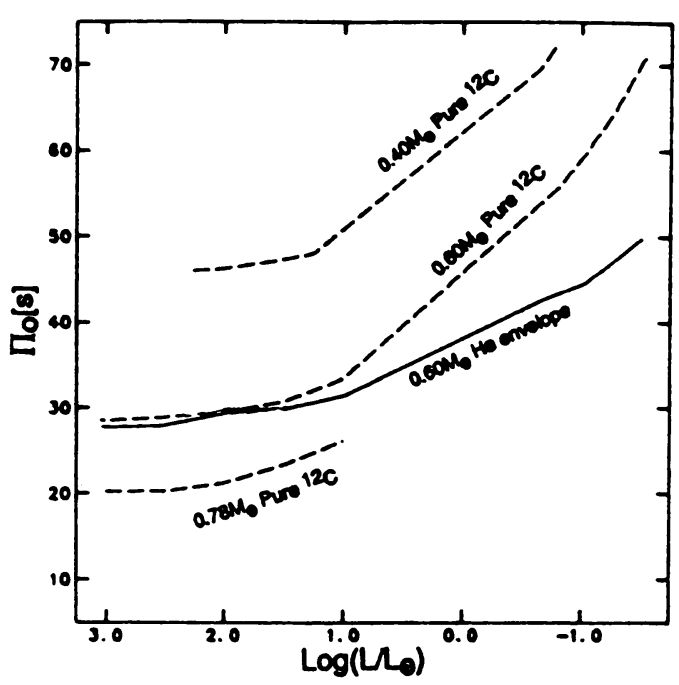

Figure 2: The characteristic period spacing as a function of stellar luminosity for various hot white dwarf models. The models are described in Kawaler et al. (1986).

Can PG1 159 be pulsating in modes that are $l=1$ and $l=3$ simultaneously? In a $0.60 \mathrm{M}_{0}$ white dwarf model with a luminosity of $100 \mathrm{~L}_{\odot}$, eight $l=3$ modes have periods that are within one second of the periods of $l=1$ modes. For these 8 model periods, the K-S test indicates values of $\Delta \Pi$ of 21.15 and 8.790 seconds, in excellent agreement with PG1159.

\section{CONCLUSIONS AND PROSPECTS}

PG1159-035 has a mass of $0.60 \mathrm{M}_{\odot}$ that is accurately determined using the uniform period spacing technique. The modes of pulsation are those with $l=3$ or $l=1$, or perhaps both. The modes we observe may be those $l=3$ and $l=1$ modes whose periods are almost identical. This could indicate nonlinear coupling between normal modes of pulsation, which acts as a mode selection mechanism. The implications of this model will be discussed in a future paper (Kawaler 1987). Additional observations of the other PG1159 variables are needed to see if they show similar mode structure. This technique should be applicable to the rich pulsation spectrum of the pulsating DB white dwarfs such as GD358, as well as to the ZZ Ceti stars (Kawaler 1987).

Kawaler, S. D., Winget, D. E., Iben, I. Jr., and Hansen, C. J. 1986, Ap. J., 302, 530.

Kawaler, S. D. 1986, Ph. D. thesis, University of Texas at Austin.

Kawaler, S. D. 1987, in preparation.

Press, W. H., Flannery, B. P., Teukolsky, S. A., and Vetterling, W. T. 1986,

Numerical Recipes (Cambridge: Cambridge University Press).

Tassoul, M. 1980, Ap. J. Suppl., 43, 469.

Winget, D. E., Kepler, S. O., Robinson, E. L., Nather, R. E., and O'Donoghue, D.1985, Ap. J., 292, 606.

Weidemann, V., and Koester, D. 1983, Astr. Ap., 121, 77. 